\title{
DETECTING PROBABLE ROCKFALLS IN OPEN PIT MINES BASED ON UAV POINT CLOUDS
}

\author{
S. M. Yousefi ${ }^{1}$, H. Arefi ${ }^{1, *}$, A. Bahroudi ${ }^{2}$ \\ ${ }^{1}$ School of Surveying and Geospatial Engineering, College of Engineering, University of Tehran, Tehran, Iran - (yousefi.1994, \\ hossein.arefi)@ut.ac.ir \\ ${ }^{2}$ School of Mining Engineering, College of Engineering, University of Tehran, Tehran, Iran - bahroudi@ut.ac.ir
}

\author{
Commission VI, WG VI/4
}

KEY WORDS: Disaster Management, Rock Face, UAV Based Processing, Open Pit Mines, Rockfall Hazard, Point Cloud Analysis

\begin{abstract}
:
Stability analysis and studying the geological features of rocks and mines have been active research topic for many years. Consequently, it is very important being prepared for probable hazards and having the ability to rescue from earth disasters, in particular in rocks and open pit mines. For this purpose, several methods have been used to measure fractures of a rock face. Among these methods are manual techniques, photogrammetric measurements, and laser scanning based techniques. With the proliferation of unmanned aerial vehicles (UAVs), these systems have been widely used in geological projects recently. Especially in the situation that the case study is very hard to be reached. In this paper, a method is developed to detect the most probable rock fall. After doing some pre-processing, RANSAC algorithm is used to fit planes to the point cloud. Then, intersections of these planes with the point cloud are computed. After some refinements on these intersections, the probable rockfalls are obtained. Point cloud analysis have some advantages over conventional image-based methods; especially in case of probable rock falls, which might be hard to detect using the rock images. However, analyzing point cloud data usually is complicated and computationally expensive.
\end{abstract}

\section{INTRODUCTION}

Photogrammetry always plays an important role in mining industry. In 1976, McCarter had taken some photographs to locate major geological structures in an open pit mine. Stability analysis of a rock vastly depends on the properties of discontinuities.

Discontinuity is a general term in rock mass engineering, and denotes any separation in a rock mass characterized by low or non-existent tensile strength (Zhang, 2016). These features are usually organized in pseudo-parallel surfaces and are referred to as joint sets or discontinuity sets ("International society for rock mechanics commission on standardization of laboratory and field tests: Suggested methods for the quantitative description of discontinuities in rock masses," 1978).

The properties of these discontinuities are geometric or nongeometric. The geometrical parameters, such as dip, dip direction, orientation, spacing, and length of a discontinuity are directly derived from rock mass exposure or bore holes made inside a rock mass using several surveying techniques (Turanboy, Ülker, \& Küçüksütçü, 2018).

The conventional method of mapping the geometric properties of discontinuities in rock exposures is based on manual surveying with compass-clinometer and measuring tape. This method is often inaccurate, dangerous and time-consuming. Nevertheless, discontinuities play a major role in controlling the geomechanical characteristics of a rock mass and thus a comprehensive assessment of their properties is crucial to the design of civil engineering works.

Remote sensing techniques offer an alternative to conventional manual surveys for assessing discontinuities in rock exposures. The obvious operational advantage is that a rock face can be mapped from a distance without any need for physical contact. Analogue and digital terrestrial photogrammetry has already been used successfully to determine discontinuity orientations.

New remote sensing techniques have several operational advantages over photogrammetry-based methods. Although the high cost of the equipment, 3D point clouds give us more control over the resolution and accuracy of the survey data and is able to deliver the survey data in near real-time. Digital photogrammetry generates survey data as a point cloud that describe the geometry of surveyed rock face with a high amount of detail and precision.

The point-cloud data can subsequently be visualised using a computer. Then data can be rotated and assessed from different points of view, and can be enhanced with colour imagery obtained with a digital camera. The scanned rock face will be inspected in detail from all directions at any time and place. Discontinuity planes and discontinuity sets are easily recognised. Experience has demonstrated that this new visual perspective allows access to a great amount of discontinuity information

\footnotetext{
* Corresponding author
} 
hidden in this data. In manual sampling of discontinuities, having direct access to the rock is inevitable which makes it often difficult and sometimes impossible to be measured. Otherwise it takes significant time to be measured considering the limitation of operator exposure to potentially unsafe conditions (Danzi, Di Crescenzo, Ramondini, \& Santo, 2012). Due to different levels of expertise of individual operators, the results of manual sampling are not reproducible. Besides, manual methods are not fast enough. So, there is a considerable need for other fast and reliable methods (Vasuki, Holden, Kovesi, \& Micklethwaite, 2014).

Thanks to digital approaches, there is a significant increase in quantity of data captured and a permanent geomechanical database has developed that can be consulted at any future time (Lemy \& Hadjigeorgiou, 2003).

However, the recent acceptance of 3D remote sensing techniques such as light detection and ranging (LiDAR) instruments, digital photogrammetry or structure from motion (SfM) (Ullman, 1979) is changing the way rock slopes are being investigated. Digital photogrammetry is a well-known technique that enables the $3 \mathrm{D}$ study of the morphology of natural and engineered rock slopes (Sturzenegger \& Stead, 2009).

Point cloud capturing these techniques usually comprise millions of points that (in this approach) are defined by their coordinates $(\mathrm{X}, \mathrm{Y}, \mathrm{Z})$ and their estimated color $(\mathrm{R}, \mathrm{G}, \mathrm{B})$.

Utilizing 3D point clouds captured from remote sensing techniques, has several advantages over image-based methods. For example, detection of possible plane fractures, which are likely to fall, is not easy using images. While visible cracks which are easy to see, are easily detected using image processing, some probable fracture plane with no sign of cracks, might be ignored, despite their risk of sliding down the rock.

In this article, a method has been proposed to detect appropriate, most probable to fall masses, in order to reduce the risk of rockslide and to apply further precautions if desired.

\section{LITERATURE REVIEW}

Discontinuity is any separation in a rock mass caused by weaknesses in a rock mass (Zhang, 2016). Each discontinuity is characterized by some features. In the following subsection, an overview of these features is given.

\subsection{Geometric discontinuity properties}

Discontinuities play an important roll in rock mass charecterisation. Particularily the geometric properties like: orientation, spacing, roughness and persistance play a key role. Especially in engineering design, these measurements are required in rock mass classification systems. The two most important features are described:

\section{a) Orientation}

Orientation is the most important geometric property, since it determines the anisotropy in mechanical behavior of the discontinuous rock mass.

The two necessary and sufficient parameters for defining a particular plane orientation are dip and dip direction (also given as strike and dip).

The dip of a particular surface is the maximum angle between the plane containing the surface and the horizontal plane.
This feature was widely measured using compass and clinometer method (Jaboyedoff et al., 2012).

Nowadays, it is easily measured using 3D point clouds, 3D laser scanning, digital stereo photogrammetry (Haneberg, 2008) and SfM (Structure from motion) (Jordá Bordehore, Riquelme, Cano, \& Tomás, 2017).

\section{b) Spacing}

Apparent spacing: The Apparent Spacing is equal to the difference in Distance values (entered in the Distance column) between adjacent joints which belong to the same joint set. This is the raw unprocessed spacing as measured directly from a linear traverse, borehole or oriented core.

True spacing: The True Spacing is the actual perpendicular spacing between adjacent joints in a joint set. The True Spacing is always less than or equal to the apparent spacing, and is calculated as follows:

$$
t=a \cos (\theta)
$$

in which, $t, a$ and $\theta$ are true spacing, apparent spacing and the angle between the pole of the mean joint set plane and the linear traverse direction, respectively.

This parameter is determined using a measuring tape, which is at least 3 meters long, a compass and a clinometer in traditionally methods. However, 3Dpoint clouds and TLS (Terrestrial Laser Scanner) and ALS (Aerial laser scanner) are used in current methods (Slob S, Turner AK, Bruining J, 2010).

Persistence, roughness, wall strength, aperture, filling, seepage, $\mathrm{N}$ of sets and block size are other parameters which are mostly used in rock mass analysis.

Although these parameters are widely accepted by the scientific and technical community, advances in new technologies and new methodologies are changing the way rock mass discontinuities are being investigated.

\subsection{Effect of joints on mechanical behavior of rock mass}

The stability of exposed rock cliffs, natural or man-made, depends to a great extent on the number and orientation of the mechanical discontinuities. Fig. 2 demonstrates the very different characteristics of a rock mass that has a single dominant mechanical discontinuity, compared to a rock mass that has three well-defined orientations of discontinuities.

The stability of exposed rock faces is thus dependent on the rock type, and the number of orientations, and characteristics of the dominant discontinuities. Intersections of discontinuities with different orientations result in rock blocks of specific sizes and shapes. However, it should be also recognized that a single mechanical discontinuity or even a very few discontinuities, oriented in particularly unfavorable attitudes might lead to major rock-slope instability or engineering geological concerns. (Bell, 1983) 

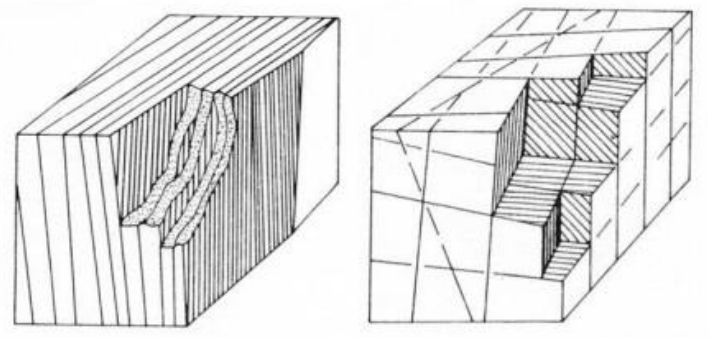

Figure 1. Effect of the number of joint sets on the mechanical behavior and appearance of a rock mass.

\section{PROPOSED METHOD}

The idea is to retrieve main planes in a rock face and find intersections. By finding nearest points to these intersections, dangerous areas (probable rockfalls) will be found. Fig. 2, shows the flowchart of the proposed method. In the following subsections, the details of each box of the flowchart are explained.

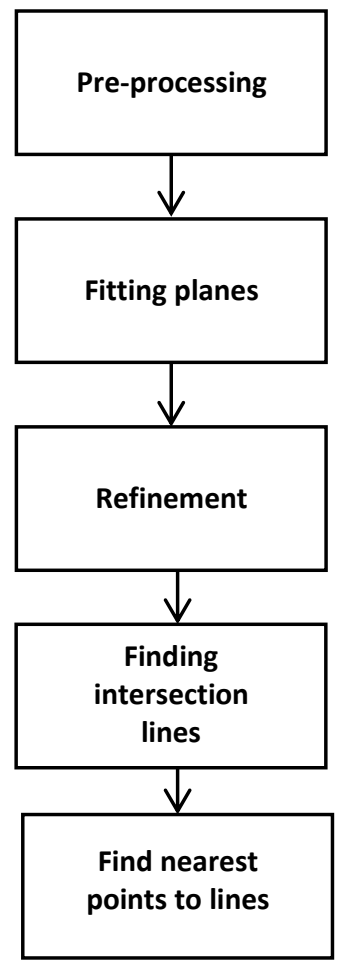

Figure 2. flowchart of the proposed method.

\subsection{Pre-processing}

First of all, some pre-processing steps are carried out on the data to omit the outliers. Before applying smoothing algorithm, the outliers are removed via statistical outlier removal (SOR) algorithm. In SOR algorithm, the average distance of each point to its $k$ nearest neighbours is computed. Then, the points with average distance larger than a multiple of standard deviation of distances are considered as outliers and subsequently removed.

Besides, the points which cannot be assigned to a plane, i.e. their distance to the nearest plane are larger than a threshold, should be considered as outliers. To determine these outlier points, distance between point and plane is computed easily and with the threshold of $0.3 \mathrm{~cm}$, points considering as noise will be deleted.

$$
f\left(X_{0}, p\right)=\frac{a x_{0}+b y_{0}+c z_{0}+d}{\sqrt{a^{2}+b^{2}+c^{2}}}
$$

In order to find principal planes, we need to smooth our surface. Poisson reconstruction method is employed to reduce unnecessary details of the surface (Bieniawski, 1998b).

The main parameter for Poisson reconstruction is octree depth. The deeper, i.e. greater, the octree level is, the finer the result will be, but also the more time and memory will be required. Octree level has been assigned 4 to 8 in this article.

To Solve the Poisson problem, it's necessary to form a vector field $\vec{V}$. We want to solve for the function $\tilde{\xi}$ such that $\nabla \tilde{\xi}=\vec{V}$.

Because of $\vec{V}$ being integrable, an exact solution does not exist. To find the best least squares approximate solution, we apply the divergence operator to form the Poisson equation.

$$
\Delta \tilde{\xi}=\nabla \cdot \vec{V}
$$

Since the Poisson algorithm is highly influenced by the noisy data, it is important to perform rectification before applying reconstruction.

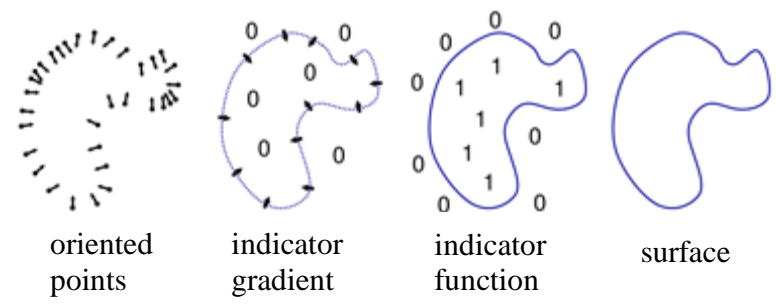

Figure 3. Extracting surface using poisson reconstruction

\subsection{Fitting Planes by Developed RANSAC}

The RANSAC shape detection algorithm, extracts 3D planes by randomly drawing minimal sets from the point data and constructing plane primitives (Bieniawski, 1998a).

In Schnabel's implementation, a standard score function that counts the number of compatible points for a shape candidate is used.

Schnabel has performed an innovative strategy for sampling. By the use of randomly sampling, shape candidates (plane, cylinder, cone, sphere and torus) are generated. The one with the highest score is computed. The best candidate is only accepted if it satisfies three constraints (Bieniawski, 1998a). If a candidate is accepted, the corresponding points of that shape, are removed from the point cloud and it continues until all shapes are detected.

Parameters: The most important parameter that vastly impress the results, is $n$ (the number of samples, i.e. support points or inliers, per primitive). The proper values for this parameter depends largely on the cloud density and the size of the planes to be detected. Other parameters of the RANSAC algorithm, are $e$ (maximum distance of an inlier point to the plane), $b$ (sampling resolution), a (maximum deviation of the planes' normal vectors). 
Table 1, shows the values used for these parameters in our simulations.

\begin{tabular}{|c|c|}
\hline Parameter & Value \\
\hline$n$ & $500-800$ \\
\hline$e$ & $0.6 \mathrm{~cm}$ \\
\hline$a$ & $25^{\circ}$ \\
\hline$b$ & 1.186 \\
\hline
\end{tabular}

Table 1. the values used for RANSAC in the simulations

Fig. 6 and Fig. 7 shows results of applying RANSAC algorithm with 700 and 500 inliers. Due to the results, number of inliers in RANSAC fitting plane, plays an important role in number of principal fracture planes detected.

\subsection{Refinement}

After applying RANSAC, plane rectification is required to eliminate the planes with the similar properties. Here, we ignore those with nearly the same normals as well as those with vertical distances shorter than the predefined threshold (here it is assigned $40 \mathrm{~cm}$ ).

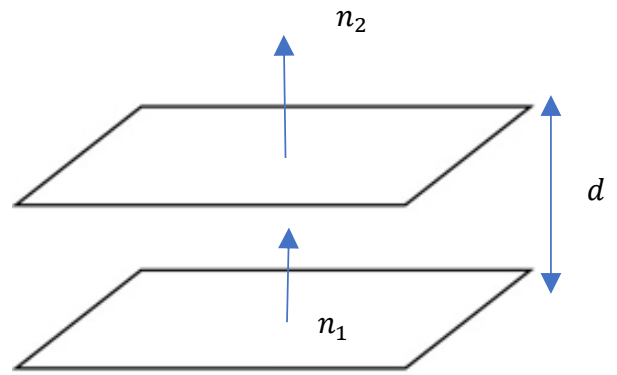

Figure 4. $\boldsymbol{n}_{\mathbf{1}}$ and $\boldsymbol{n}_{\mathbf{2}}$ are nearly the same but vertical distance of these planes is large. So, these two planes are different and none of them should be deleted.

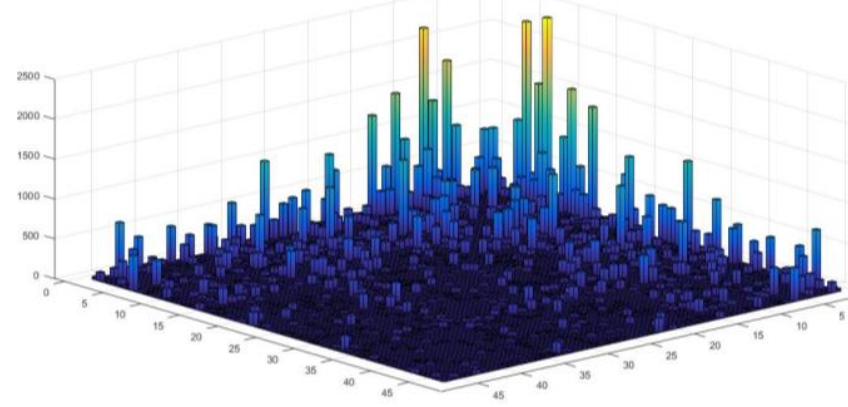

Figure 5. histogram of points lying on two corresponding planes in 85 planes' algorithm.

Considering the octree level 8 and extracting 85 planes, the histogram of common points between two planes is shown in Fig.5. As can be seen, the histogram is symmetric and after the refinement step, there is just 50 planes left.

\subsection{Finding intersection lines}

Now the question is how we can find the fractures. After preparing the planes, intersections of them can easily be found. However, these intersections need to be related to the proper points in point cloud.

Considering $N$ planes, for each point a sorted distance vector is computed. $\boldsymbol{d}_{i}$, the distance vector related to the $i$ th point, is a $1 \times$ $N$ vector whose elements are sorted distances of the $i$ th point to the planes $\left(d_{i, 1} \leq d_{i, 2} \leq \cdots \leq d_{i, N}\right)$.

$$
R_{i, j}=\frac{d_{i, j}}{d_{i, j+1}}
$$

$R_{i, j}$ is the ratio of two distances; distance of the $i_{t h}$ point from $j_{\text {th }}$ plane and the distance of that point from $j+1_{t h}$ plane.

Consider $k_{t h}$ point lying on a plane, $R_{k, 1}$ will be very small. If it lies on two planes, this variable will be very close to one. We should consider that $d_{i, 1}$ is the distance of $i_{t h}$ point to its nearest plane.

Fitting lines: A line has been extracted from each group of intersection points. However, after applying removing noise algorithm, some part of the case study becomes very sparse, thus small fractures might not be detected as we expected. Intersections that have more than 300 points are plotted in Fig. 6.

\section{RESULTS}

The method depends highly on the number of planes which is defined by an expert. Here, 85, 12, 10, 9 and 8 planes' approach was applied. The final results of 9 and 12 planes approach are shown in Fig.6 and Fig.7. Although the difference between these two figures cannot be negligible, a meaningful difference is not found.

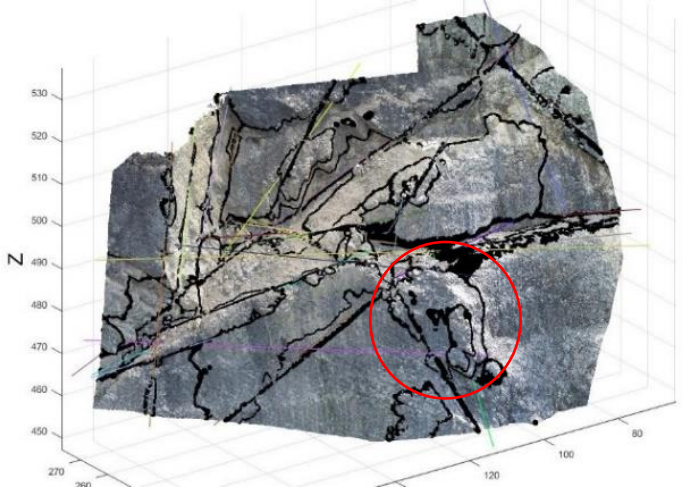

Figure 6. Intersection of 9 planes with the point cloud, considering 700 inliers in RANSAC algorithm. 


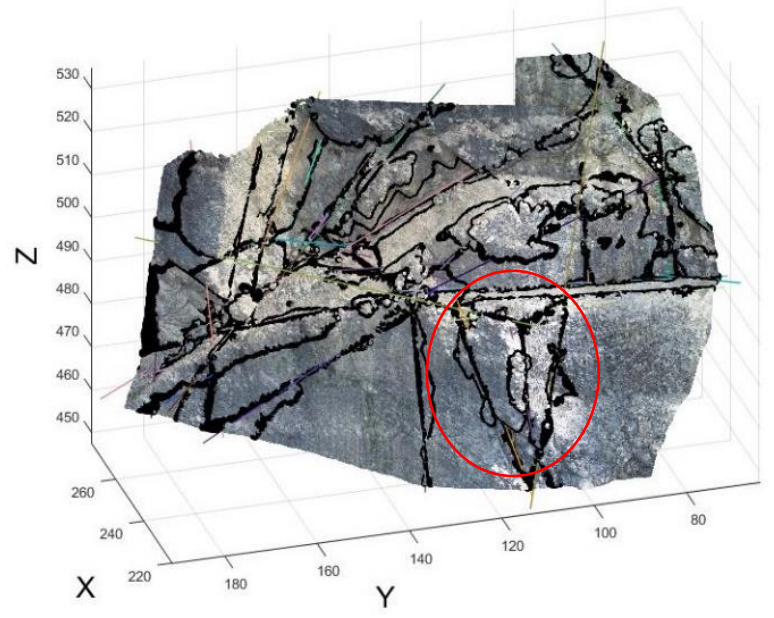

Figure 7. Intersection of 12 planes with the point cloud, considering 500 inliers in RANSAC algorithm.

Black points in the Fig. 6 are due to the intersection of the main fracture planes and the point cloud. Obviously to show more detailed results we have to change our parameters.

As you can see in both Fig. 6 and Fig. 7, there is a red circle which identifies the intersection of three planes and a rock mass is located there. Therefore, the rock mass has a high potential of falling down.

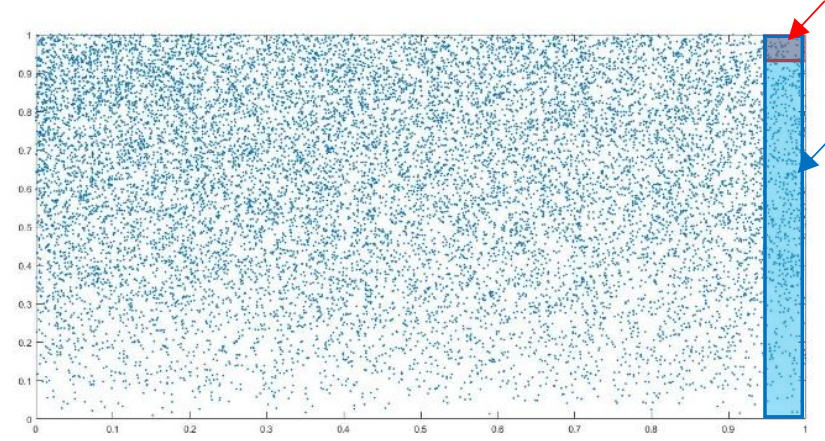

Figure 8. Blue and red rectangles shows the points that are probably on the intersection of two and three planes, respectively.

Blue rectangle in Fig. 8 shows the points that are probably on the intersection of two planes, because the distance between these points and their two closest planes, are nearly the same.

Points surrounded with red rectangle in Fig. 8 are probably on the intersection of three planes, because their distance to their first nearest planes are close to each other.

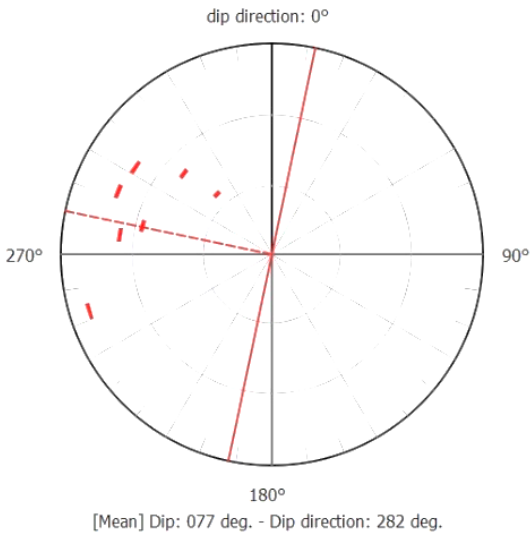

Figure 9. Stereographic projection of structural planes, detected by applying 700 inliers in RANSAC.

As shown in Fig. 9, mean DIP and mean DIP direction of structural planes are $77^{\circ} \mathrm{C}$ (centigrade) and $282^{\circ} \mathrm{C}$. Main steps in this stereogram is $30.00^{\circ} \mathrm{C}$ with resolution of $5^{\circ} \mathrm{C}$.

\section{CONCLUSIONS}

The primary focus of this paper is the development and use of semi-automated procedure to extract the geometric properties of discontinuities encountered in rock exposures via analysis of point-cloud datasets. The point-cloud datasets offer opportunities to obtain much improved geometric characterisations of discontinuities within a rock mass, compared to discontinuity characterisations resulting from conventional field mapping methods. Yet the characteristics of the point-cloud datasets, including their relatively large size and lack of spatial organisation, present difficulties in analysing them efficiently and extracting the desired geometric information.

Nowadays, with the proliferation of UAVs, this method is one of most used equipment in digital photogrammetry. Therefore, it is possible to speed up external procedures specially in high cliffs and those sites which are not reachable nor safe to investigate. The method proposed in this paper, is a powerful tool for digitizing fracture planes faster and in some cases with a lower cost. Reliability of results are indeed higher than those in manual methods.

The most important advantage of the proposed method, is its capability of measuring under the inaccessibly circumstances. Besides, high-resolution data, captured from UAVs, can be still used in 3D modelling and further applications if needed.

In contrast with image-based techniques, the proposed method, is able to detect unexpected cracks, which may not be easily seen on rock images.

Although it is encouraging, the variety of rocks morphology in the sites lead us to many further tests to be sure about roughness of the algorithm. There is always a big concern about the level of automation of finding cracks in a rockface, too. In this work it is needed to set some parameters on visual inspection of an expert.

To conclude, the method mentioned above is suitable for measuring high and steep slopes that people cannot reach and slopes that contain large numbers of joints.

\section{REFERENCES}

Bell, F. G. (1983). Fundamentals of engineering geology. Butterworths. 
Bieniawski, Z. T. (1998a). Efficient RANSAC for Point-Cloud Shape Detection: Computer Graphics Bonn. In Computer Graphics Forum (Vol. https://doi.org/10.1111/j.1467-8659.2007.01016

Bieniawski, Z. T. (1998b). Poisson Surface Reconstruction. In Eurographics Symposium on Geometry Processing. New York: John Wiley \& Sons.

Danzi, M., Di Crescenzo, G., Ramondini, M., \& Santo, A. (2013). Use of unmanned aerial vehicles (UAVs) for photogrammetric surveys in rockfall instability studies. Rendiconti Online Societa Geologica Italiana, 24, 82-85.

Haneberg, W. C. (2008). Using close range terrestrial digital photogrammetry for 3-D rock slope modeling and discontinuity mapping in the United States. Bulletin of Engineering Geology and the Environment, 67(4), 457469. https://doi.org/10.1007/s10064-008-0157-y

International society for rock mechanics commission on standardization of laboratory and field tests: Suggested methods for the quantitative description of discontinuities in rock masses. (1978). International Journal of Rock Mechanics and Mining Sciences \& Geomechanics Abstracts, 15(6), 319-368. https://doi.org/10.1016/01489062(78)91472-9

Jaboyedoff, M., Oppikofer, T., Abellán, A., Derron, M.-H., Loye, A., Metzger, R., \& Pedrazzini, A. (2012). Use of LIDAR in landslide investigations: a review. Natural Hazards, 61(1), 5-28. https://doi.org/10.1007/s11069-010-9634-2

Jordá Bordehore, L., Riquelme, A., Cano, M., \& Tomás, R. (2017). Comparing manual and remote sensing field discontinuity collection used in kinematic stability assessment of failed rock slopes. International Journal of Rock Mechanics and Mining Sciences, 97, 24-32. https://doi.org/10.1016/j.ijrmms.2017.06.004

Lemy, F., \& Hadjigeorgiou, J. (2003). Discontinuity Trace Map Construction Using Photographs of Rock Exposures. Rock Mechanics \& Mining Sciences, 40, 903-917.

Slob S, Turner AK, Bruining J, H. H. (2010). Automated rock mass characterisation using 3-D terrestrial laser scanning. Retrieved from https://webapps.itc.utwente.nl/librarywww/papers_2010/ phd/slob.pdf

Sturzenegger, M., \& Stead, D. (2009). Close-range terrestrial digital photogrammetry and terrestrial laser scanning for discontinuity characterization on rock cuts. Engineering Geology, 106(3-4), 163-182. https://doi.org/10.1016/J.ENGGEO.2009.03.004

Turanboy, A., Ülker, E., \& Küçüksütçü, C. B. (2018). A new approach for assessing stability of rock slopes considering centroids of weak zones. Journal of Mining \& Environment, 9(1), 1-18.

Ullman, S. (1979). The interpretation of visual motion. Massachusetts Inst of Technology Pr.

Vasuki, Y., Holden, E.-J., Kovesi, P., \& Micklethwaite, S. (2014). Semi-automatic mapping of geological Structures using UAV-based photogrammetric data: An image analysis approach. Computers\&Geosciences, 69, 22-32.

Zhang, L. (2016). Engineering properties of rocks. Retrieved from https://arizona.pure.elsevier.com/en/publications/enginee ring-properties-of-rocks-second-edition 